$$
\begin{aligned}
& \text { UNITU_THEP 94-2 } \\
& \text { SW } 9411
\end{aligned}
$$

\title{
Self-consistent solution to a complex fermion determinant with space dependent fields ${ }^{\dagger}$
}

\author{
U. Zückert, R. Alkofer and H. Weigel ${ }^{\ddagger}$ \\ Institute of Theoretical Physics \#, Tübingen University, \\ Auf der Morgenstelle 14, D-72076 Tübingen, Germany
}

March 4, 1994

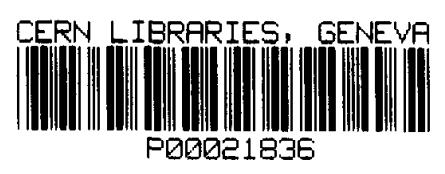

\begin{abstract}
A numerical routine is provided which allows to compute and minimize a mesonic energy functional involving a complex fermion determinant. The energy functional is evaluated by summing over the real and imaginary parts of the eigenvalues of a non-Hermitian oneparticle Dirac Hamiltonian. In order to minimize the energy functional the corresponding eigenfunctions are used to set up the equations of motion for the meson fields. Iteration of these equations yields the self-consistent configuration of the meson fields.
\end{abstract}

${ }^{\dagger}$ Supported by the Deutsche Forschungsgemeinschaft (DFG) under contract Re 856/2-1.

$\ddagger$ Supported by a Habilitanden-scholarship of the DFG.

\# E-mail to: zueckert@mailserv.zdv.uni-tuebingen.de

UNITU-THEP-2/1994

Submitted to: Computer Physics Communications. 


\section{PROGRAM SUMMARY}

Title of the program: vecscal

Catalogue number:

Program obtainable from: CPC Program Library, Queen's University of Belfast, N. Ireland (see application form in this issue).

Computer: UNIX WORKSTATIONS: DEC-5000; HP-9000.

Installation: Institute of Theoretical Physics, Tübingen University.

Operating system: UNIX.

Programming language used: FORTRAN 77.

High speed storage required:

DEC: $2.392 \mathrm{MB}$ data and stack segments, $2.076 \mathrm{MB}$ real memory;

HP: $1.022 \mathrm{MB}$ virtual size;

Peripherals used: standard input, standard output, disk

Leywords: Nambu-Jona-Lasinio model, chiral soliton, hedgehog ansatz, complex functional determinant, static energy functional.

Nature of the physical problem

Quark fields are integrated out from a generalized Nambu-Jona-Lasinio model yielding a complex functional determinant which involves composite meson fields. This determinant is evaluated for static but space-dependent meson field configurations. The configuration which minimizes the corresponding energy functional is constructed.

Method of solution

A basis for the quark spinors suited for spherically symmetric configurations is provided. The one-particle Dirac Hamiltonian involving space dependent meson fields is diagonalized using this basis. Special to this case is the non-hermiticity of the Hamiltonian. The fermion determinant is calculated by summing over the resulting complex eigenvalues. The corresponding eigenfunctions serve to set up the equations of motion for the meson fields. The solution to these equations is obtained by iteration.

Restriction of the complexity of the problem

Typical running time

The running time strongly depends on the size of the momentum space basis as well as the number of lattice points. Typical parameters yield for a single iteration $92 \mathrm{~min}$ (DEC), 
19.5min (HP) cpu-time.

Unusual features of the program

Access to the IMSL library is needed[1].

References

[1] IMSL, Inc. (1989), IMSL MATH/LIBRARY User's Manual, version 1.1, IMSL, Houston.

\section{LONG WRITE UP}

\section{The physical problem}

The purpose of this program is to construct the self-consistent soliton configuration of an extended Nambu-Jona-Lasinio (NJL) model[1, 2]. This program represents a generalization of another routine[3] wherein only one dynamical field is considered[4,5]. This solution represents the key ingredient for the description of baryons as solitonic excitations in the framework of the N.JL model with (axial-) vector mesons included[6]-[9].

As the starting point we assume the bosonized version[2] of the two flavor NJL model with scalar $(S)$, pseudoscalar $(P)$, vector $(V)$ and axial-vector $(A)$ interactions. The corresponding action splits into a mesonic part

$$
\mathcal{A}_{m}=\int d^{4} x\left(-\frac{1}{4 g_{1}} \operatorname{tr}\left(M^{\dagger} M-m^{0}\left(M+M^{\dagger}\right)+\left(m^{0}\right)^{2}\right)+\frac{1}{4 g_{2}} \operatorname{tr}\left(V_{\mu} V^{\mu}+A_{\mu} A^{\mu}\right)\right) .
$$

and a fermion determinant

$$
\mathcal{A}_{F}=\operatorname{Tr} \log (i \not D)=\operatorname{Tr} \log \left(i \not \partial+V+\gamma_{5} A-\left(P_{R} M+P_{L} M^{\dagger}\right)\right)
$$

such that $\mathcal{A}_{N J L}=\mathcal{A}_{F}+\mathcal{A}_{m}$. The scalar and pseudoscalar fields are contained in $M=S+i P$. Here $g_{i}$ and $m^{0}$ denote the NJL coupling constants and the current quark mass, respectively. $P_{R, L}$ are projectors on right- and left-handed quark spinors. Since the NJL model is not renormalizable a regularization prescription has to be imposed. Here we adopt Schwinger's proper time description[10]. Then one more parameter, the cut-off $\Lambda$, is introduced. In order to perform this regularization it is necessary to continue $\mathcal{A}_{F}$ to Euclidean space $\left(x_{0}=\right.$ $\left.-i \tau, V_{0}=-i V^{4}, A_{0}=-i A^{4}\right)$ yielding $\mathcal{A}_{F}^{(E)}=\operatorname{Tr} \log \left(i D_{E}\right)$. For the problem at hand $\mathcal{A}_{F}^{(E)}=$ $\mathcal{A}_{R}+A_{I}$ is complex. The proper time regularization corresponds to substituting the logarithm 
by a parameter integral

$$
\begin{aligned}
& \mathcal{A}_{R}=\frac{1}{2} \operatorname{Tr} \log \left(\not_{E}^{\dagger} \mathbb{D}_{E}\right) \longrightarrow-\frac{1}{2} \int_{1 / \Lambda^{2}}^{\infty} \frac{d s}{s} \operatorname{Tr} \exp \left(-s \not D E_{E}^{\dagger} \mathbb{D}_{E}\right) \\
& \mathcal{A}_{I}=\frac{1}{2} \operatorname{Tr} \log \left(\left(\not_{E}^{\dagger}\right)^{-1} \not D_{E}\right) \longrightarrow-\frac{1}{2} \int_{1 / \Lambda^{2}}^{\infty} \frac{d s}{s} \operatorname{Tr} \exp \left(-s\left(\not D_{E}^{\dagger}\right)^{-1} \not_{E}\right) .
\end{aligned}
$$

Note that $\mathcal{A}_{I}$ stays finite for $\Lambda \rightarrow \infty$.

Upon variation it can be shown that only $S$ acquires a space-time independent, nonvanishing vacuum expectation value $\langle S\rangle=m \mathbf{1}[2]$. The constituent quark mass $m$ is the solution to the Schwinger-Dyson equation[5]

$$
m=m^{0}+m^{3} \frac{N_{C} g_{1}}{2 \pi^{2}} \Gamma\left(-1,\left(\frac{m}{\Lambda}\right)^{2}\right)
$$

where $N_{C}=3$ denotes the number of colors. Performing a derivative expansion[2] of $\mathcal{A}_{R}$ allows to relate the parameters of the model to physical quantities

$$
m_{\pi}^{2}=\frac{m^{0} m}{g_{1} f_{\pi}^{2}}, \quad f_{\pi}^{2}=\frac{6 m^{2}}{g_{V}^{2}} \frac{1}{1+6 m^{2} / m_{\rho}^{2}} \quad \text { and } \quad m_{\rho}^{2}=\frac{g_{V}^{2}}{4 g_{2}}
$$

with the vector coupling constant given by

$$
g_{v}=\left(\frac{1}{8 \pi^{2}} \Gamma\left(0, \frac{m^{2}}{\Lambda^{2}}\right)\right)^{-1 / 2} .
$$

As inputs the pion mass $\left(m_{\pi}\right)$ and decay constant $\left(f_{\pi}\right)$ as well as the $\rho$ meson mass $\left(m_{\rho}\right)$ are used. Chosing the constituent quark mass $m$ completely fixes all parameters.

As usual we assume the static hedgehog ansat $z$ for the fields involved. This introduces six radial functions $\Phi(r), \Theta(r), \omega(r), G(r), F(r)$ and $H(r)$ :

$$
\begin{aligned}
M(\boldsymbol{r}) & =m \Phi(r) \exp (i \boldsymbol{\tau} \cdot \hat{\mathbf{r}} \Theta(r)) \\
V_{\mu}^{0} & =\omega(r) \delta_{\mu 4} \\
V_{4}^{a} & =0, \quad V_{i}^{a}=\epsilon^{a k i} \hat{r}^{k} G(r), \\
A_{4}^{a} & =0, \quad A_{i}^{a}=\hat{r}^{i} \hat{r}^{a} F(r)+\delta^{i a} H(r)
\end{aligned}
$$

while all other field components vanish. For the ongoing discussion the radial functions in eqn.(8) will generically be labeled $\varphi$. The ansat $z$ (8) leads to a one-particle Dirac Hamiltonian

$$
\begin{aligned}
h= & \boldsymbol{\alpha} \cdot \boldsymbol{p}+i \omega(r)+m \boldsymbol{\Phi}(r) \beta\left(\cos \Theta(r)+i \gamma_{5} \boldsymbol{\tau} \cdot \hat{\mathbf{r}} \sin \Theta(r)\right) \\
& +\frac{1}{2}(\boldsymbol{\alpha} \times \hat{\mathbf{r}}) \cdot \boldsymbol{\tau} G(r)+\frac{1}{2}(\boldsymbol{\sigma} \cdot \hat{\mathbf{r}})(\boldsymbol{\tau} \cdot \hat{\mathbf{r}}) F(r)+\frac{1}{2}(\boldsymbol{\sigma} \cdot \boldsymbol{\tau}) H(r)
\end{aligned}
$$

which enters the Euclidean Dirac operator

$$
i \beta \not D_{E}=-\partial_{\tau}-h .
$$

Obviously $h$ is not Hermitian since $\omega(r)$ is real causing the eigenvalues $\epsilon_{\mu}=\epsilon_{\mu}^{R}+i \epsilon_{\mu}^{I}$ to be complex. Therefore one has to distinguish between left and right eigenstates

$$
h|\mu\rangle=\epsilon_{\mu}|\mu\rangle \quad\langle\tilde{\mu}| h=\epsilon_{\mu}\langle\tilde{\mu}| \quad \text { i.e. } h^{\dagger}|\tilde{\mu}\rangle=\epsilon_{\mu}^{*}|\tilde{\mu}\rangle .
$$


These states are normalized to $\langle\dot{\mu} \mid \nu\rangle=\delta_{\mu \nu}$.

When the functional traces in $(3,4)$ are carried out an energy functional in Minkowski space can be extracted $[6],[8],[9]$

$$
E[\varphi]=E_{\mathrm{val}}^{R}+E_{\mathrm{val}}^{I}+E_{\mathrm{vac}}^{R}+E_{\mathrm{vac}}^{I}+E_{\mathrm{m}}-E_{\mathrm{vac}}^{R}\left[\varphi=\varphi_{\mathrm{vac}}\right]
$$

where the contribution from the trivial vacuum has been subtracted. The various pieces in eqn.(12) read

$$
\begin{array}{rlrl}
E_{\mathrm{vac}}^{R}= & \frac{N_{C}}{4 \sqrt{\pi}} \sum_{\nu}\left|\epsilon_{\nu}^{R}\right| \Gamma\left(-\frac{1}{2},\left(\epsilon_{\nu}^{R} / \Lambda\right)^{2}\right), & E_{\mathrm{val}}^{R}=N_{C} \eta_{\mathrm{val}} \epsilon_{\mathrm{val}}^{R} \\
E_{\mathrm{vac}}^{I}= & \frac{-N_{C}}{2 \sqrt{\pi}} \sum_{\nu} \epsilon_{\nu}^{I} \operatorname{sign}\left(\epsilon_{\nu}^{R}\right) \Gamma\left(\frac{1}{2},\left(\epsilon_{\nu}^{R} / \Lambda\right)^{2}\right), & E_{\mathrm{val}}^{I}=N_{C} \eta_{\mathrm{val}} \epsilon_{\mathrm{val}}^{I} \\
E_{\mathrm{m}}= & 4 \pi \int d r r^{2}\left(\frac{m_{\pi}^{2} f_{\pi}^{2}}{2 m^{0}}\left[m(\Phi(r)-1)+2 m^{0} \Phi(r)(1-\cos \Theta(r))\right]\right. \\
& & \left.+\left(\frac{m_{\rho}}{g_{V}}\right)^{2}\left(G^{2}(r)+\frac{1}{2} F^{2}(r)+F(r) H(r)+\frac{3}{2} H^{2}(r)-2\left(\frac{m_{\omega}}{m_{\rho}}\right)^{2} \omega^{2}(r)\right)\right) .
\end{array}
$$

$\epsilon_{\mathrm{val}}$ denotes the energy eigenvalue of the bound valence quark and $\eta_{\mathrm{val}}=0(1)$ for $\epsilon_{\mathrm{val}}<(>) 0$. $E_{\mathrm{m}}$ denotes the mesonic contribution ${ }^{1}$ which is obtained from $\mathcal{A}_{\mathrm{m}}$ (1). Extremizing the total energy (12) yields the equations of motion

$$
0=\frac{\delta E}{\delta \varphi}=\frac{\delta E_{m}}{\delta \varphi}+\sum_{\kappa=R, I} \sum_{\mu} \frac{\partial\left(E_{\mathrm{val}}^{R}+E_{\mathrm{val}}^{I}+E_{\mathrm{vac}}^{R}+E_{\mathrm{vac}}^{I}\right)}{\partial \epsilon_{\mu}^{\kappa}} \frac{\delta \epsilon_{\mu}^{\kappa}}{\delta \varphi} .
$$

In order to extract the functional dependence of $\epsilon_{\mu}^{\kappa}$ it is helpful to notice that the nonHermitian Dirac operator may be decomposed into an Hermitian and anti-Hermitian part[8]

$$
h=h_{\Theta}+i \omega
$$

where $h_{\Theta}$ includes all Hermitian terms of the Euclidean Dirac Hamiltonian (9). In a basis where $h_{\Theta}$ and $\omega$ are both real and symmetric this separation yields the relation $|\dot{\nu}\rangle=\left|\nu^{*}\right\rangle$. It furthermore allows to write

$$
\begin{aligned}
\epsilon_{\nu}^{R} & =\left\langle\Psi_{\nu}^{R}\left|h_{\Theta}\right| \Psi_{\nu}^{R}\right\rangle-\left\langle\Psi_{\nu}^{I}\left|h_{\Theta}\right| \Psi_{\nu}^{I}\right\rangle-\left\langle\Psi_{\nu}^{I}|\omega| \Psi_{\nu}^{R}\right\rangle-\left\langle\Psi_{\nu}^{R}|\omega| \Psi_{\nu}^{I}\right\rangle, \\
\epsilon_{\nu}^{I} & =\left\langle\Psi_{\nu}^{R}|\omega| \Psi_{\nu}^{R}\right\rangle-\left\langle\Psi_{\nu}^{I}|\omega| \Psi_{\nu}^{I}\right\rangle+\left\langle\Psi_{\nu}^{I}\left|h_{\Theta}\right| \Psi_{\nu}^{R}\right\rangle+\left\langle\Psi_{\nu}^{R}\left|h_{\Theta}\right| \Psi_{\nu}^{I}\right\rangle
\end{aligned}
$$

where we employed the decomposition $|\nu\rangle=\left|\Psi_{\nu}^{R}\right\rangle+i\left|\Psi_{\nu}^{I}\right\rangle$. Note also that $\left\langle\nu^{*}\right|=\left\langle\Psi_{\nu}^{R}\right|+i\left\langle\Psi_{\nu}^{I}\right|$. From eqn.(18) the functional derivatives needed in (16) are apparent. It is appropriate to define densities ${ }^{2}$

$$
\begin{aligned}
\rho(\boldsymbol{x}, \boldsymbol{y}) & =N_{c}\left\{\rho^{\mathrm{val}}(\boldsymbol{x}, \boldsymbol{y})+\rho^{\mathrm{vac}}(\boldsymbol{x}, \boldsymbol{y})\right\} \quad \text { and } \quad b(\boldsymbol{x}, \boldsymbol{y})=N_{c}\left\{b^{\mathrm{val}}(\boldsymbol{x}, \boldsymbol{y})+b^{\mathrm{vac}}(\boldsymbol{x}, \boldsymbol{y})\right\} \\
\rho^{\mathrm{val}}(\boldsymbol{x}, \boldsymbol{y}) & =\eta_{\text {val }}\left\{\Re \psi_{\text {val }}(\boldsymbol{x}) \bar{\psi}_{\text {val }}(\boldsymbol{y})+\Im \psi_{\text {val }}(\boldsymbol{x}) \bar{\psi}_{\text {val }}(\boldsymbol{y})\right\} \\
\rho^{\mathrm{vac}}(\boldsymbol{x}, \boldsymbol{y}) & =\sum_{\nu}\left\{f_{R}\left(\epsilon_{\nu} / \Lambda\right) \Re \psi_{\nu}(\boldsymbol{x}) \bar{\psi}_{\nu}(\boldsymbol{y})+f_{I}\left(\epsilon_{\nu} / \Lambda\right) \Im \psi_{\nu}(\boldsymbol{x}) \bar{\psi}_{\nu}(\boldsymbol{y})\right\} \\
b^{\mathrm{val}}(\boldsymbol{x}, \boldsymbol{y}) & =\eta_{\text {val }}\left\{\Re \psi_{\text {val }}(\boldsymbol{x}) \psi_{\text {val }}^{\dagger}(\boldsymbol{y})-\Im \psi_{\text {val }}(\boldsymbol{x}) \psi_{\text {val }}^{\dagger}(\boldsymbol{y})\right\} \\
b^{\text {vac }}(\boldsymbol{x}, \boldsymbol{y}) & =\sum_{\nu}\left\{f_{I}\left(\epsilon_{\nu} / \Lambda\right) \Re \psi_{\nu}(\boldsymbol{x}) \psi_{\nu}^{\dagger}(\boldsymbol{y})-f_{R}\left(\epsilon_{\nu} / \Lambda\right) \Im \psi_{\nu}(\boldsymbol{x}) \psi_{\nu}^{\dagger}(\boldsymbol{y})\right\} .
\end{aligned}
$$

\footnotetext{
${ }^{1}$ We allow for $m_{\omega} \neq m_{\rho}$ although isospin symmetry implies $m_{\omega^{\prime}}=m_{\rho}$.

${ }^{2} \psi_{\nu}(\boldsymbol{x})$ represents the coordinate space representation of $|\nu\rangle$.
} 
Real $(\Re)$ and imaginary $(\Im)$ parts refer to the expansion coefficients of the free basis $(c f$. section 2). The regulator functions

$$
\begin{aligned}
& f_{R}\left(\epsilon_{\nu} / \Lambda\right)=-\frac{1}{2 \sqrt{\pi}} \operatorname{sign}\left(\epsilon_{\nu}^{R}\right) \Gamma\left(\frac{1}{2},\left(\epsilon_{\nu}^{R} / \Lambda\right)^{2}\right)+\frac{1}{\sqrt{\pi}}\left(\epsilon_{\nu}^{I} / \Lambda\right) \exp \left(-\left(\epsilon_{\nu}^{R} / \Lambda\right)^{2}\right) \\
& f_{I}\left(\epsilon_{\nu} / \Lambda\right)=-\frac{1}{2 \sqrt{\pi}} \operatorname{sign}\left(\epsilon_{\nu}^{R}\right) \Gamma\left(\frac{1}{2},\left(\epsilon_{\nu}^{R} / \Lambda\right)^{2}\right)
\end{aligned}
$$

reflect the derivative of the energy functional with respect to the energy eigenvalues. The specific form of the equations of motion (16) becomes

$$
\begin{aligned}
\Phi(r) & =\frac{m^{0}}{m} \cos \Theta(r)-\frac{m^{0}}{4 \pi m_{\pi}^{2} f_{\pi}^{2}} \operatorname{tr} \int \frac{d \Omega}{4 \pi}\left(\cos \Theta(r)+i \gamma_{5} \boldsymbol{\tau} \cdot \hat{\mathbf{r}} \sin \Theta(r)\right) \rho(\boldsymbol{x}, \boldsymbol{x}) \\
\sin \Theta(r) & =\frac{m}{m_{\pi}^{2} f_{\pi}^{2}} \operatorname{tr} \int \frac{d \Omega}{4 \pi}\left(\sin \Theta(r)-i \gamma_{5} \boldsymbol{\tau} \cdot \hat{\mathbf{r}} \cos \Theta(r)\right) \rho(\boldsymbol{x}, \boldsymbol{x}) \\
\omega(r) & =\frac{g_{V}^{2}}{4 m_{\rho}^{2}} \operatorname{tr} \int \frac{d \Omega}{4 \pi} b(\boldsymbol{x}, \boldsymbol{x}) \\
G(r) & =-\frac{g_{V}^{2}}{4 m_{\rho}^{2}} \operatorname{tr} \int \frac{d \Omega}{4 \pi}((\boldsymbol{\gamma} \times \hat{\mathbf{r}}) \cdot \boldsymbol{\tau}) \rho(\boldsymbol{x}, \boldsymbol{x}) \\
F(r) & =-\frac{g_{V}^{2}}{4 m_{\rho}^{2}} \operatorname{tr} \int \frac{d \Omega}{4 \pi} \beta(3(\boldsymbol{\sigma} \cdot \hat{\mathbf{r}})(\boldsymbol{\tau} \cdot \hat{\mathbf{r}})-(\boldsymbol{\sigma} \cdot \boldsymbol{\tau})) \rho(\boldsymbol{x}, \boldsymbol{x}) \\
H(r) & =\frac{g_{V}^{2}}{4 m_{\rho}^{2}} \operatorname{tr} \int \frac{d \Omega}{4 \pi} \beta((\boldsymbol{\sigma} \cdot \hat{\mathbf{r}})(\boldsymbol{\tau} \cdot \hat{\mathbf{r}})-(\boldsymbol{\sigma} \cdot \boldsymbol{\tau})) \rho(\boldsymbol{x}, \boldsymbol{x}) .
\end{aligned}
$$

Here the trace is taken with respect to Dirac and flavor indices only. Note that $\operatorname{tr} \int d \Omega b(\boldsymbol{r}, \boldsymbol{r})$ is the baryon charge density.

\section{$2 \quad$ Numerical methods}

\subsection{Eigenstates of the static Hamiltonian}

In order to solve for the Hamiltonian a suitable basis has to be chosen. The hedgehog ansatz has the celebrated feature to commute with the grand spin operator

$$
G=J+\frac{\tau}{2}=\boldsymbol{l}+\frac{\sigma}{2}+\frac{\tau}{2}
$$

which is the sum of the total spin $\boldsymbol{J}$ and the isospin $\boldsymbol{\tau} / 2$. The spin itself is decomposed into orbital angular momentum $\boldsymbol{l}$ and intrinsic spin $\boldsymbol{\sigma} / 2$. Then the eigenstates are also eigenstates of $G^{2} \hat{=} G(G+1)$ and $G_{3} \hat{=} M$. These eigenstates are constructed as linear combinations of the eigenstates $|\mu\rangle^{0}$ of the free Hamiltonian $\left(\varphi=\varphi_{\mathrm{vac}}\right)$

$$
\begin{aligned}
& \left|1, n, j=G+\frac{1}{2}, M\right\rangle=\mathcal{N}_{n}^{G}\left(\begin{array}{c}
i w_{n G}^{+} j_{G}\left(k_{n G} r\right)\left|G G+\frac{1}{2} G M\right\rangle \\
w_{n G}^{-} j_{G+1}\left(k_{n G} r\right)\left|G+1 G+\frac{1}{2} G M\right\rangle
\end{array}\right), \\
& \left|2, n \cdot j=G-\frac{1}{2}, M\right\rangle=\mathcal{N}_{n}^{G}\left(\begin{array}{c}
i w_{n G}^{+} j_{G}\left(k_{n G} r\right)\left|G G-\frac{1}{2} G M\right\rangle \\
-w_{n G}^{-} j_{G-1}\left(k_{n G} r\right)\left|G-1 G-\frac{1}{2} G M\right\rangle
\end{array}\right),
\end{aligned}
$$




$$
\begin{aligned}
& \left|3, n, j=G+\frac{1}{2}, M\right\rangle=\mathcal{N}_{n}^{G}\left(\begin{array}{c}
i w_{n G}^{+} j_{G+1}\left(k_{n G} r\right)\left|G+1 G+\frac{1}{2} G M\right\rangle \\
-w_{n G}^{-} j_{G}\left(k_{n G} r\right)\left|G G+\frac{1}{2} G M\right\rangle
\end{array}\right), \\
& \left|4, n, j=G-\frac{1}{2}, M\right\rangle=\mathcal{N}_{n}^{G}\left(\begin{array}{c}
i w_{n G}^{+} j_{G-1}\left(k_{n G} r\right)\left|G-1 G-\frac{1}{2} G M\right\rangle \\
w_{n G}^{-} j_{G}\left(k_{n G} r\right)\left|G G-\frac{1}{2} G M\right\rangle
\end{array}\right) .
\end{aligned}
$$

Here $j_{l}$ denote the spherical Bessel functions. $|l j G M\rangle$ represent states where first the orbital angular momentum $l$ and the spin are coupled to the total angular momentum $j=l \pm 1 / 2$ and secondly isospin and $j$ are coupled to the grand spin $G=j \pm 1 / 2 . M$ is the projection of $G$. This coupling scheme yields the selection rules

$$
j= \begin{cases}G+1 / 2, & l=\left\{\begin{array}{l}
G+1 \\
G
\end{array}\right. \\
G-1 / 2, & l=\left\{\begin{array}{l}
G \\
G-1
\end{array} .\right.\end{cases}
$$

This system is treated in a spherical box of finite radius $D$. Thereby the momentum eigenvalues are discretized by demanding the components with $l=G$ to vanish at the boundary[11] i.e.

$$
j_{l}\left(k_{n l} D\right)=0
$$

Thus the energy eigenvalues of the free Hamiltonian $h\left(\varphi=\varphi_{\mathrm{vac}}\right)$ are given by $e^{0}=E_{n G}=$ $\pm \sqrt{k_{n G}^{2}+m^{2}}$. The $\epsilon^{0}$ 's are needed for the subtraction of $E_{\mathrm{vac}}\left[\varphi=\varphi_{\mathrm{vac}}\right]$ in eqn. (12). The kinematical factors $w_{n l}^{ \pm}$are defined as

$$
w_{n l}^{+}=\sqrt{1+m / E_{n l}}, \quad w_{n l}^{-}=\operatorname{sign}\left(E_{n l}\right) \sqrt{1-m / E_{n l}} .
$$

while the normalization factor is given by

$$
\mathcal{N}_{n}^{-l}=\left[D^{3 / 2}\left|j_{l+1}\left(k_{n l} D\right)\right|\right]^{-1}
$$

The numerical diagonalization of $h$ requires an upper bound, $k_{\max }$ for the momenta $k_{n l}$ leaving for each $l$ a finite number of momentum eigenstates $N(l): k_{1 l}, \ldots, k_{N(l) l}$. Taking into account that for each momentum one has two energy eigenvalues it is obvious that we have $2 N(l)$ eigenfunctions of $h\left(\varphi=\varphi_{\mathrm{vac}}\right)$ for each grand spinor in eqn.(29).

Next the matrix elements of $h$ for $\varphi \neq \varphi_{\text {vac }}$ have to be computed. Due to the grand spin symmetry these are diagonal in $G$ and independent of $M$. For the explicit form of these matrix elements we refer to appendix $A$ of ref.[8]. Here we only wish to remark that states with different intrinsic parity $\pi$ do not mix. In eqn.(29) states 1 and 2 carry $\pi=+$, while 3 and 4 are characterized by $\pi=-$. In each channel $(G, \pi)$ the Hamiltonian corresponds to a $4 N(G) \times 4 N(G)$ dimensional non-Hermitian matrix. Diagonalization yields the eigenenergies ${ }^{3}$ $\epsilon_{G}^{ \pm}(\mu)$ and the corresponding eigenvectors $V_{G}^{( \pm)}(n, \mu)$ with $(\mu=1, \ldots, 4 N(G))$. Here it is important to note that both $\epsilon_{G}^{ \pm}(\mu)$ and $V_{G}^{( \pm)}(n, \mu)$ are complex. The eigenvectores are used to define complex radial functions $g_{\mu}^{(G, \pm, i)}$ and $f_{\mu}^{(G, \pm, i)}(i=1,2)$ appearing in the coordinate space representation of the eigenstates of $h$

$$
\begin{aligned}
& \Psi_{\mu}^{(G,+)}=\left(\begin{array}{c}
i g_{\mu}^{(G,+; 1)}(r)\left|G G+\frac{1}{2} G M\right\rangle \\
f_{\mu}^{(G,+; 1)}(r)\left|G+1 G+\frac{1}{2} G M\right\rangle
\end{array}\right)+\left(\begin{array}{c}
i g_{\mu}^{(G,+; 2)}(r)\left|G G-\frac{1}{2} G M\right\rangle \\
-f_{\mu}^{(G,+; 2)}(r)\left|G-1 G-\frac{1}{2} G M\right\rangle
\end{array}\right) \\
& \Psi_{\mu}^{(G,-)}=\left(\begin{array}{c}
i g_{\mu}^{(G,-; 1)}(r)\left|G+1 G+\frac{1}{2} G M\right\rangle \\
-f_{\mu}^{(G,-; 1)}(r)\left|G G+\frac{1}{2} G M\right\rangle
\end{array}\right)+\left(\begin{array}{c}
i g_{\mu}^{(G,-; 2)}(r)\left|G-1 G-\frac{1}{2} G M\right\rangle \\
f_{\mu}^{(G,-; 2)}(r)\left|G G-\frac{1}{2} G M\right\rangle
\end{array}\right) .
\end{aligned}
$$

\footnotetext{
${ }^{3}$ The upper index refers to intrinsic parity.
} 
For example we have

$$
g_{\mu}^{(G,+; 1)}(r)=\sum_{n=1}^{2 N(G)} V_{G}^{(+)}(n, \mu) \mathcal{N}_{n}^{G} w_{n G}^{+} j_{G}\left(k_{n G} r\right) .
$$

The traces appearing in the equations of motion (22-27) are then computed with the help of the matrix elements listed in appendix A of ref.[8]. Again we only present one generic example

$$
\begin{aligned}
& \operatorname{tr} \int d \Omega \Psi_{\mu}^{(G,+)}(\boldsymbol{x}) \bar{\Psi}_{\mu}^{(G,+)}(\boldsymbol{x}) i_{\gamma_{5}} \tau \cdot \hat{\mathbf{r}}=2\left(g_{\mu}^{(G,+; 1)}(r) f_{\mu}^{(G,+; 1)}(r)+g_{\mu}^{(G,+; 2)}(r) f_{\mu}^{(G,+; 2)}(r)\right) \\
&+4 \sqrt{G(G+1)}\left(g_{\mu}^{(G,+; 1)}(r) f_{\mu}^{(G,+; 2)}(r)-g_{\mu}^{(G,+; 2)}(r) f_{\mu}^{(G,+; 1)}(r)\right) .
\end{aligned}
$$

Note that no sum over $\mu$ is implied.

The incomplete $\Gamma$ functions with half-integer arguments appearing $e . g$. in the energy functional $(13,14)$ or the regulator functions $(20,21)$ are computed by relating them to the complementary error function

$$
\operatorname{erfc}(|x|)=\int_{x^{2}}^{\infty} \frac{d s}{\sqrt{\pi s}} e^{-s}=\frac{1}{\sqrt{\pi}} \Gamma\left(\frac{1}{2}, x^{2}\right) .
$$

Incomplete $\Gamma$ functions with half-integer arguments other than $1 / 2$ are related to the complementary error function via integration by parts.

In order to finally make the equations suitable for the iterative procedure we have to care about finite size $(D<\infty)$ effects. From the Dirac and flavor structure of the operators in eqns.(23-27) it is obvious that in continuum limit $(D \rightarrow \infty)$ the baryon number zero $(B=0)$ sector allows for the trivial solution that all fields except of $\Phi$ are zero. Eqn.(22) becomes the analogue of the Schwinger-Dyson equation(5) and thus $\Phi=1$. Due to the restricted basis at finite $D$ the trivial configuration is not necessarily reproduced in the $B=0$ sector ${ }^{4}$. Thus corresponding subtractions have to be made at each step of the iteration. For the boundary conditions chosen here this concerns eqns.(22) and (25). Let us denote the solutions for finite $D$ in the $B=0$ sector to these equations by $\Phi_{0}(r)$ and $G_{0}(r)$, respectively. As can be seen from figure B.2 of ref.[12] $\Phi_{0}(r)$ deviates significantly from unity in the vicinity of $r=D$. In the $B=1$ sector we then evaluate the matrix elements of the Hamiltonian using the ratio $\Phi(r) / \Phi_{0}(r)$ where $\Phi(r)$ is the solution of (22). Also $G_{0}(r)$ is subtracted from (25). Using an alternative set of boundary conditions this problem cannot be avoided but is merely transferred to other equations.

Furthermore the $\omega$ meson profile assumes a non-vanishing value proportional to $1 / D^{3}$ at large distance. This behavior has no dramatic influence on the soliton mass. However, higher moments of the baryon density ( $\epsilon . g$. the isoscalar radius) might diverge in the continuum limit $D \rightarrow \infty$. Therefore the energy functional is supplemented by a constraint

$$
E[\varphi] \longrightarrow E[\varphi]+\lambda \int d^{3} r f_{\epsilon}(r) \omega^{2}(r)
$$

wherein $f_{\epsilon}(r)$ is a positive radial function which vanishes everywhere except within a small vicinity $\epsilon$ of $r=D$. By iteration of the modified equations of motions $\lambda$ is adjusted such that the additional term vanishes. Of course, there is some arbitrariness in choosing $f_{\epsilon}(r)$. We demand the change of the total energy with $\lambda$ included to deviate from the case $\lambda=0$ by less than $1 \mathrm{MeV}$.

\footnotetext{
${ }^{4}$ At finite $D$ the functional unity is not completely reproduced.
} 


\section{Description of the program}

The purpose of the program is to calculate the energy (12) and the meson profiles $\{\varphi\}=$ $\Phi, \Theta, \omega, G, F$ and $H$ which characterize the soliton solution to the NJL model with vector mesons. This is achieved by diagonalizing the Hamiltonian (9) in the basis (29) for an initial guess of $\varphi$. Subsequently the equations of motion (22-27) are employed to iterate $\varphi$.

\subsection{The main program}

The program starts by reading the initial parameters from standard input. The number of iterations is chosen as an input quantity (and not by some condition) because the numbers of iterations needed very strongly depends on the quantities to be calculated. E.g. the energy or mass of the soliton converges very quickly, and a few iterations are sufficient, whereas the accurate determination of the eigenfunctions requires dozens of iterations. The mesh size, the number of points where the field is iterated, the value of $k_{\max }$ in units of the physical cut-off $\Lambda$ and the box size are additional inputs. Furthermore, the constituent quark mass, the pion decay constant $\left(f_{\pi}\right)$, the meson masses $\left(m_{\pi}, m_{\rho}\right.$ and $\left.m_{\omega}\right)$ and an initial guess for the profile functions $\varphi(r)$ are provided. In a first calculation the cut-off $\Lambda$ and the current quark mass $m^{0}$ are determined. The program also allows to use a different cut-off $\Lambda_{I}$ for the imaginary part of the action (4,14 and 21). This value might eventually be set to "infinity" since this part of the action turns out to be finite. Furthermore the coupling constants are computed from the input quantities. Next the correction $\Phi_{0}(r)$ to the scalar field is evaluated and the "constraint function" $f_{\epsilon}(r)(39)$ is defined.

The program is organized in a way that every channel with given grand spin and parity channel is treated independently. Additionally, the grand spin zero cases have to be treated separately as only the state carrying total angular momentum $j=G+1 / 2=1 / 2$ exists. First, the momenta and energies of the free basis states are computed. Then the required Bessel functions are calculated and stored in arrays. The complex matrix elements in a given channel are calculated with a Simpson integration and diagonalized using the IMSL routine devccg[13]. The eigenvectors are normalized and used to construct the radial functions

$g_{\mu}^{(G,+, 1)}(r)$, etc. defined in eqns.(34-36). In the next step the contribution of this channel to the densities appearing in the equations of motion (22-27) and the correction to the $\rho$ meson field, $G_{0}(r)$ are calculated. Also the contribution to the vacuum parts of the energy (13 and 14 ) is added. In the grand spin zero and positive parity channel the valence quark state is identified and $\eta_{\text {val }}$ is set according to the sign of the valence quark energy.

If no states with momenta less than $k_{\max }$ are left the total energy is computed. The meson fields are updated according to the equations of motion (22-27) at all points of the lattice.

Finally the axial vector coupling constant of the nucleon is evaluated by use of the current field identity[2],[8].

The results are written to standard output and eventually a new iteration is performed.

\subsection{Subroutines and functions}

Subroutine devceg

IMSL subroutine which diagonalizes a complex matrix[13]. Subroutine ortho 
Normalizes the complex eigenvectors $V_{G}^{( \pm)}(n, \mu)$.

Functions $f f r$ and $f f i$

Calculate the regulator functions for the equations of motion $(20,21)$.

Functions eregr and eregi

Calculate the contribution of an energy eigenvalue to the vacuum part of the energy.

Function $e r f c c$

Calculates the complementary error function $\operatorname{erfc}(x)$. Cf. chapter 6.2 in ref.[14].

Function simps

Calculates radial integrals for the Hamiltonian matrix.

Function rtbis

Identifies the discretized momenta $k_{n l}$.

Function $y$

Calculates Bessel functions $j_{l}(z)$.

Subroutine smid

Calculates correction field for the chiral radius $\Phi_{0}$.

Subroutine null

Calculates zeros needed in the subroutine smid.

Function $g h$

Calculates the kernel of the Bethe-Salpeter equation for free pions.

Function $d g$

Derivative of $g h$ with respect to $(m / \Lambda)^{2}$.

Function gamma0

Calculates the incomplete $\Gamma$-function $\Gamma(0, x)$.

\section{Testing the program}

Trivial tests establishing the independence of the lattice size $\Delta r$, the box radius $D$ or the upper bound for the momentum states $k_{\max }$ have, of course, been performed.

In the case that the imaginary part of the action is not regularized $\left(\Lambda_{I} \rightarrow \infty\right)$ we have verified that the baryon number is unity.

We have also tested the current field identities which provide two distinguished possibilities to evaluate $g_{A}$. Numerically the deviation has been found to be less than $1 \%$.

We have furthermore observed that the mass of the soliton decreases monotonically at each step of the iteration procedure until the minimum is reached.

\section{Acknowledgements}

We gratefully acknowledge the valuable collaboration with $\mathrm{H}$. Reinhardt. We are also thankful for helpful hints by J. Schlienz regarding the treatment of the scalar field. 


\section{References}

[1] Y. Nambu and G. Jona-Lasinio, Phys. Rev. 122 (1961) 345; 124 (1961) 246.

[2] D. Ebert and H. Reinhardt, Nucl. Phys. B271 (1986) 188.

[3] R. Alkofer and H. Weigel, "Self-consistent solution to a fermion determinant with space dependent fields". Submitted to Computer Physics Communications.

[4] H. Reinhardt, Nucl. Phys. A503 (1989) 825.

[5] H. Reinhardt and R. Wünsch, Phys. Lett. B215 (1988) 577; Phys. Lett. B230 (1989) 93 ;

T. Meissner, F. Grümmer and K. Goeke, Phys. Lett. B227 (1989) 296;

R. Alkofer, Phys. Lett. B236 (1990) 310.

[6] R. Alkofer, H. Reinhardt, H. Weigel and U. Zückert, Phys. Lett. B298 (1993) 132.

[7] R. Alkofer, H. Reinhardt, H. Weigel and U. Zückert, Phys. Rev. Lett. 69 (1992) 1874;

C. Schüren, F. Döring, E. Ruiz Arriola and K. Goeke, Nucl. Phys. A565 (1993) 687.

[8] U. Zückert, R. Alkofer, H. Reinhardt and H. Weigel, "The Chiral Soliton of the NambuJona-Lasinio Model with Vector and Axial Vector Mesons", HEP-PH/9303271, Nucl. Phys. A to be published.

[9] U. Zückert, R. Alkofer, H. Reinhardt and H. Weigel, in preparation.

[10] J. Schwinger, Phys. Rev. 82 (19.51) 664.

[11] S. Kahana and G. Ripka, Nucl. Phys. A429 (1984) 462.

[12] H. Weigel, R. Alkofer and H. Reinhardt, Nucl. Phys. B387 (1992) 638.

[13] IMSL, Inc. (1989), IMSL MATH/LIBRARY User's Manual, version 1.1, IMSL, Houston.

[14] W. A. Press, B. P. Flannery, S. A. Teukolsky and W. T. Vetterling, "Numerical Recipes", Cambridge University Press 1989. 
